\title{
Cerclage Location and Gestational Age at Delivery
}

\author{
Fatima Estrada, MD ${ }^{1}$ Scarlett Karakash, $\mathrm{MD}^{2}$ Terry SeeToe, $\mathrm{MD}^{3} \quad$ Jeremy Weedon, $\mathrm{PhD}^{3}$ \\ Howard Minkoff, MD ${ }^{1}$ \\ ${ }^{1}$ Departments of Obstetrics and Gynecology, Maimonides Medical \\ Center, Brooklyn, New York \\ ${ }^{2}$ Division of Maternal-Fetal Medicine, Department of Obstetrics and \\ Gynecology, Stanford University, Stanford, California \\ ${ }^{3}$ Division of Research, State University of New York, New York, New York

\begin{abstract}
Address for correspondence Fatima Adelaida Maria Estrada Trejo, MD, Division of Maternal Fetal Medicine, Department of Obstetrics, Gynecology, and Women's Health, Montefiore Medical Center/Jacobi Medical Center/Albert Einstein College of Medicine, 50 Patricia Lane, Unit 412, Bronx, NY 10465 (e-mail: festrada.trejo@gmail.com).
\end{abstract}

Am J Perinatol Rep 2019;9:e195-e199.

\begin{abstract}
\section{Keywords}

- preterm delivery

- cervical cerclage

- cervical length

- cerclage height

- proximal residual length

- history indicated cerclage

- physical exam indicated cerclage

- ultrasound indicated cerclage

Objective Multiple authors have suggested cerclage position is a determinant of "success." We assessed the interaction between cervical length $(\mathrm{CL})$, cerclage height (cerH), proximal residual length (PRL), gestational age at delivery, and rate of delivery $\leq 34$ weeks, in this study.

Study Design Present study is a retrospective cohort study of all cerclages placed at Maimonides Medical Center from 2006 to 2016. Outcomes: gestational age at delivery and delivery before 34 weeks; predictors: PRL, cerH, CL; and indications for cerclage: history $(\mathrm{Hx})$, physical exam (PE), and ultrasound (US) indicated cerclage. A general linear model was used to predict power-transformed age at delivery from cerH, $\mathrm{CL}$, and indication for cerclage. Subanalyses by indication were conducted. Logistic regression was used for delivery $\leq 34$ weeks.

Results The cerH by indication did not reach statistical significance $(p=0.090)$. When stratified by indications, the effect of cerH on age at delivery was apparent for $\mathrm{Hx}$ (adjusted $R^{2}=0.18, p<0.001$ ) and PE (adjusted $R^{2}=0.43, p=0.004$ ) cerclages but not for US cerclages (adjusted $R^{2}=0.08, p=0.206$ ). Logistic regression predicting delivery $\leq 34$ weeks $(n=29)$ produced similar results.

Conclusions For $\mathrm{Hx}$ and $\mathrm{PE}$ indicated cerclages, the location of the stitch may influence the timing of delivery. Specifically, the higher the cerclage, the more advanced the gestational age at delivery.
\end{abstract}

Preterm birth, defined as a delivery between $20^{0 / 7}$ and $36^{6 / 7}$ weeks, is the leading cause of neonatal morbidity and mortality in the United States. ${ }^{1}$ The rate of extreme prematurity in the U.S. has remained unchanged despite a decrease in late preterm deliveries. $^{2}$

There are multiple etiologies of spontaneous preterm delivery, including preterm labor, preterm premature rupture of membranes, and cervical insufficiency. The latter, whose pathophysiology is still poorly understood, is the inability of the uterine cervix to retain a pregnancy in the absence of clinical signs and symptoms of labor; and its diagnosis is made based on a history of painless cervical dilation after the first

received

July 26, 2018 accepted after revision March 14, 2019
DOI https://doi.org/

10.1055/s-0039-1688778. ISSN 2157-6998. trimester with subsequent expulsion of the pregnancy in the second trimester. ${ }^{3}$

Several nonsurgical and surgical modalities have been proposed for treatment of cervical insufficiency. Cerclage placement, being one of them, may be indicated based on a patient's history (history of two or more second trimester losses or history of cerclage placement), physical examination findings (open cervix on physical exam), or a history of preterm birth and findings of short cervix on ultrasound (less than $2.5 \mathrm{~cm}$ ). ${ }^{3}$

The original descriptions for cerclage placement emphasized placing the suture "as high as possible to approximate the level of the internal cervical os." 4 Multiple studies have suggested
Copyright $\odot 2019$ by Thieme Medical Publishers, Inc., 333 Seventh Avenue, New York, NY 10001, USA. Tel: +1(212) 584-4662.
License terms

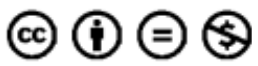


that differences in cerclage position may contribute to the variability observed in cerclage efficacy. ${ }^{5}$ One example is the work of Guzman et al who performed a precerclage measurement and a postcerclage measurement and then found and association between an upper cervical length $(\mathrm{CL})<10 \mathrm{~mm}$ and delivery before 36 weeks of gestation; however, unlike our study, only patients with physical exam indicated cerclage were included. ${ }^{6}$ Other authors have suggested that in the case of ultrasound indicated cerclage, differences in cerclage position may influence the time from cerclage placement until delivery. ${ }^{7}$

No prior research has looked into the relationship of cerclage height (cerH: length from the cerclage to the external cervical os), proximal residual length (PRL: length of cervix that remains closed proximal to the cerclage), and $\mathrm{CL}$ (distance from external to internal cervical os) after cerclage placement ( - Fig. 1), to gestational age at delivery and rate of preterm delivery ( $<34$ weeks) for all types of cerclages segregated based on indication. ${ }^{8}$ With this in mind, we studied the relationship of $\mathrm{CL}$, cerH, and PRL to the gestational age at delivery and rate of delivery at less than 34 weeks for cerclages placed by any of American College of Obstetricians and Gynecologists' (ACOG) recommended indications (history, physical exam, and ultrasound).

By studying the effect of cerclage position in all indicated cerclages separated by indication, we expected to differentiate if cerclage position would affect efficacy in some but not all cerclages.

\section{Methods}

We performed a historical cohort study that included all patients who had a cerclage placed at Maimonides Medical Center (MMC), and who were subsequently followed at the hospital's perinatal diagnostic center, from 2006 to 2016. Exclusion criteria were age younger than 18 years, multiple gestation, known uterine anomaly at the time of cerclage placement, history of cervical excision procedure, iatrogenic preterm delivery $(<37$ weeks), cerclage placement for indications other than those recommended by ACOG, as well as a lack of delivery information and/or pre- and postcerclage cervical measurements.

All data were downloaded from the hospital's ultrasound and coding program (ASOBGYN [AS software for Obstetrics and Gynecology]). Every chart coded under "cerclage" or "short cervix" was reviewed ( $n=1,167)$. Of these, 379 had a cerclage placed during the index pregnancy. Among those, a total of 267

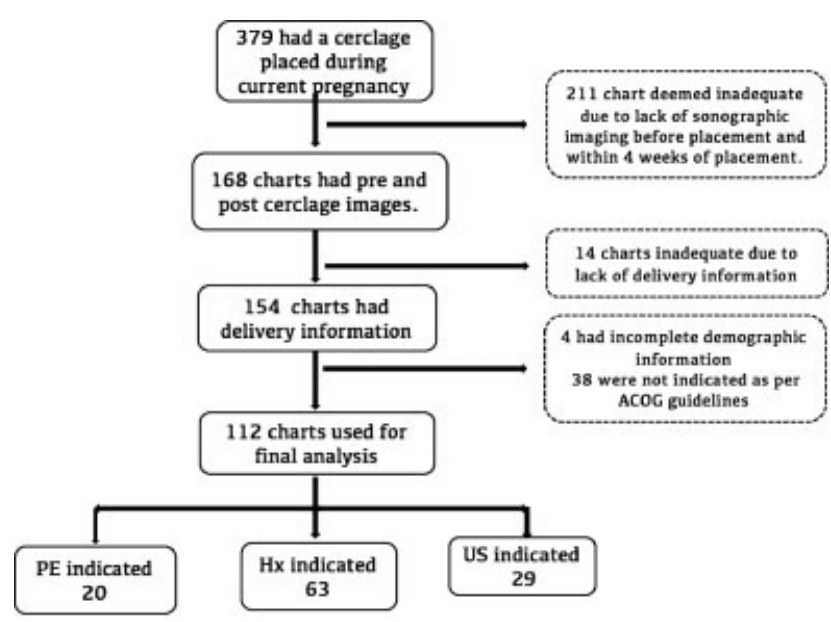

Fig. 2 Total charts reviewed and total number of charts used for analysis. ACOG, American College of Obstetrics and Gynecologists; $\mathrm{Hx}$, history; PE, physical exam; US, ultrasound.

patients were excluded either because they lacked sonographic records before or after placement $(n=211)$, delivery information $(n=14)$, such as gestational age at delivery or indications for delivery (e.g., iatrogenic or spontaneous), had the cerclages placed for an indication other than the ones approved by ACOG guidelines $(n=38)$, or had incomplete demographic information $(n=4)$. The remaining 112 patients were used for analysis (-Fig. 1).

As per hospital protocol, routine CL screening is performed in every patient at the time of anatomy ultrasound to diagnose patients with a short cervix (CL of less than or equal to $2.5 \mathrm{~cm}$ ). For patients at increased risk of preterm delivery (history of preterm delivery, history of second trimester loss, or history of cerclage placement in prior pregnancy) screening starts at 14 to 16 weeks of gestation. We considered the CL measurements prior to (and in the case of multiple reads, the most proximate to) the procedure the precerclage $\mathrm{CL}$, and we used those scans to confirm the indication for placement. We performed postcerclage measurements between 1 and 4 weeks after the cerclage placement.

We used the standardized method of obtaining $\mathrm{CL}$ as described by Berghella ${ }^{8}$ and as recommended by the cervical length education and review (CLEAR) program. The images collected between 2006 and 2016 at the perinatal unit and saved in ASOBGYN software were used for the measurement of cerH, CL, and PRL (-Fig. 2). Given differences in the

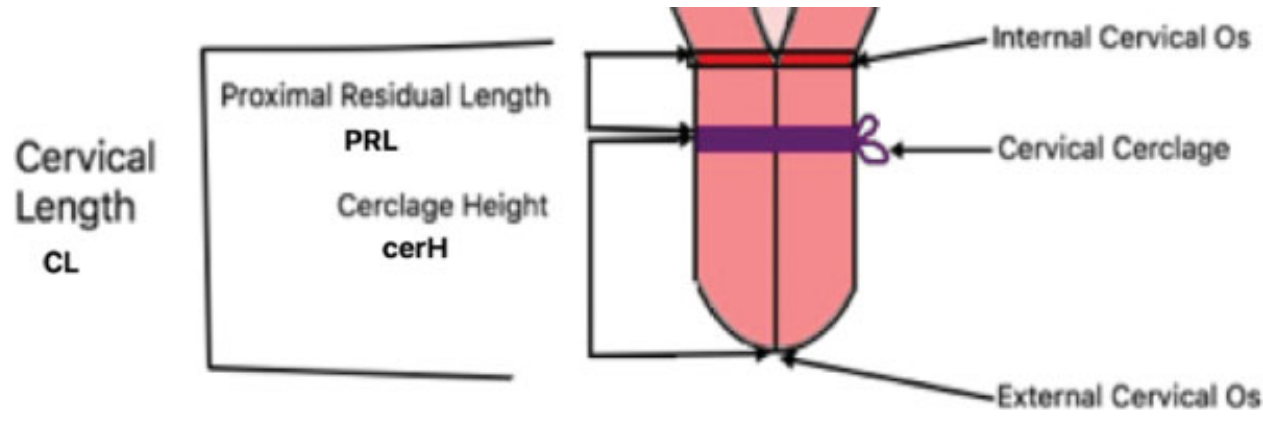

Fig. 1 The diagram of CL, cerH and PRL. CL, cervical length; cerH, cerclage height; PRL, proximal residual length. 


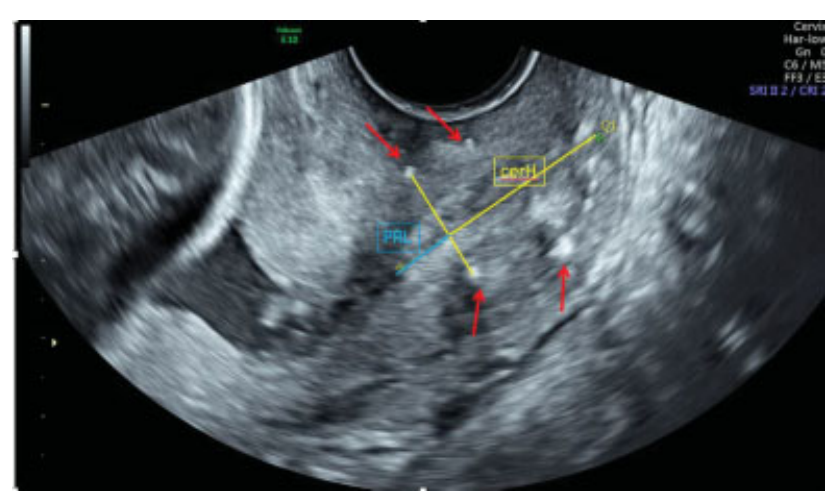

Fig. 3 PRL in blue, cerH in yellow, and red arrows are pointing toward the stitches for a 2 knot cerclage. cerH, cerclage height; PRL, proximal residual length;

number of sutures ( $n=$ one or two) placed, the stitch closest to the internal os was used for measurement of the previously defined lengths ( - Fig. $\mathbf{3}$ ).

The indications for cerclage were history of cervical insufficiency (history of one or more second trimester losses or history of cerclage placement; $n=63$ ), physical examination findings (open cervix on physical exam; $n=20$ ) or a history of preterm birth (at less than 34 weeks of gestation) in conjunction with findings of short cervix on ultrasound (less than $2.5 \mathrm{~cm} ; n=29$ ).

In the initial analysis cerH, CL, and PRL were looked at as predictors of delivery time. However, it was found that cerH and PRL were too tightly linked to be analyzed separately. A general linear model (GLM) was used to predict power-transformed age at delivery (AD) from cerH, $\mathrm{CL}$, and indication for cerclage (IC); interactions among these terms were investigated, as were the possible utility of polynomial terms in cerH and CL. Subanalyses were then conducted and stratified by the indication for cerclage. Model residuals were inspected for skew and for outliers. Adjusted $R^{2}$ values (i.e., corrected for the over-fitting problem) are reported as measures of strength of association. Significance level was set at 0.05 . In a secondary analysis, logistic regression was used to predict delivery $\leq 34$ weeks, in the same manner as above; the Nagelkerke's pseudo $R^{2}$ statistic is reported. The Hosmer-Lemeshow lack of fit test was applied.

\section{Results}

One hundred and twelve patients were included in the final analysis. These were broken down by indication for cerclage: history indicated ( $n=63)$, physical exam indicated $(n=20)$, and ultrasound indicated ( $n=29$; - Fig. 2). In the GLM analysis including all cerclage indication types, a significant CL by indication for cerclage $(p=0.034)$ was detected; there was also a significant indication for cerclage main effect $(p=0.003)$.

The cerH by indication for cerclage did not reach statistical significance $(p=0.090)$. However, when stratified by the different indications, the effect of cerH on age at delivery was apparent for history indicated (adjusted $R^{2}=0.18$, $p<0.001$ ) and physical exam indicated (adjusted $R^{2}=0.43$, $p=0.004$ ) cerclages but not for ultrasound indicated cerclages (adjusted $R^{2}=0.08, p=0.206$ ). Though there was statistical significance for the history indicated group, the adjusted $R^{2}$ value suggests that the strength of the association is not high. For cases, where the indication for cerclage is a physical exam, the evidence (based on a small subsample) that age at delivery is positively associated with cerH was more robust but still modest. For ultrasound indicated cerclages, the association is much weaker. CL was not a significant predictor in any model. -Fig. 4 shows for each indication, the curvilinear regression

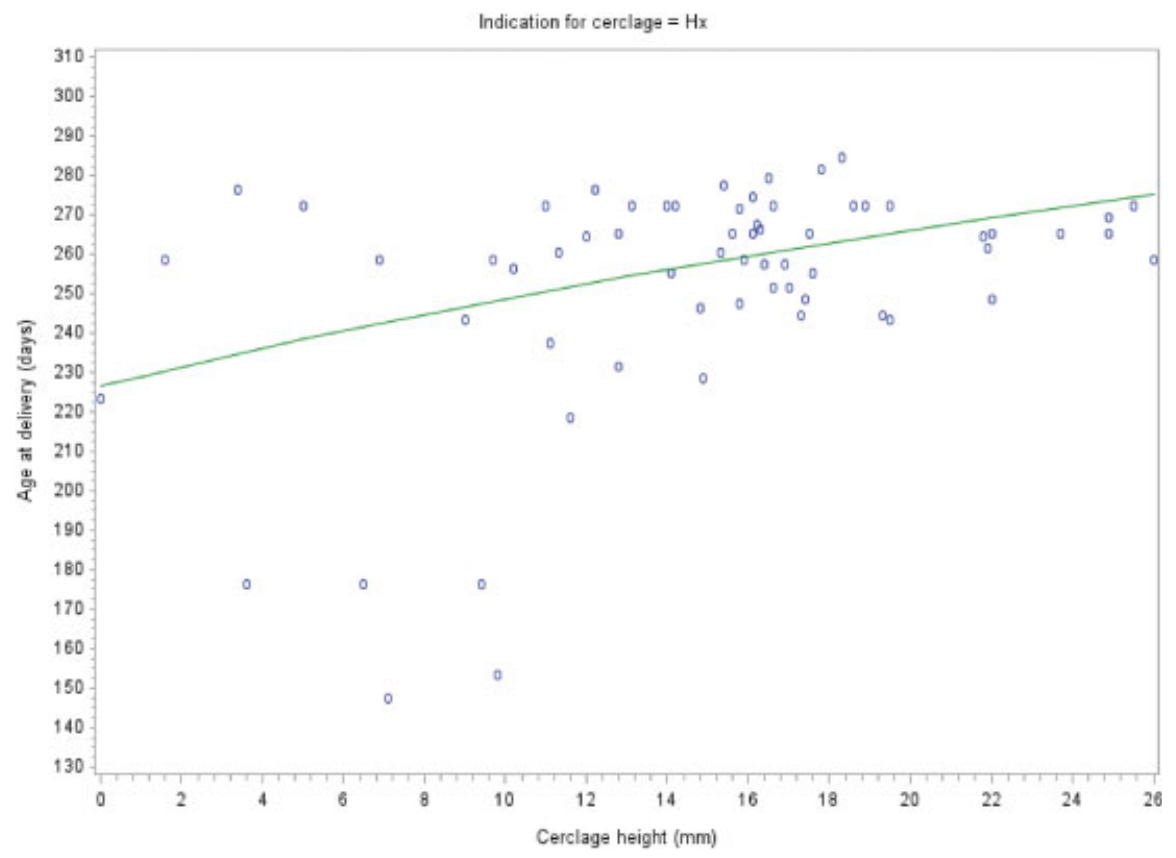

Fig. 4 The curvilinear regression function (adjusted for mean cervical length for cases with that indication), overlaid over raw data points for Physical exam indicated cerclages. 


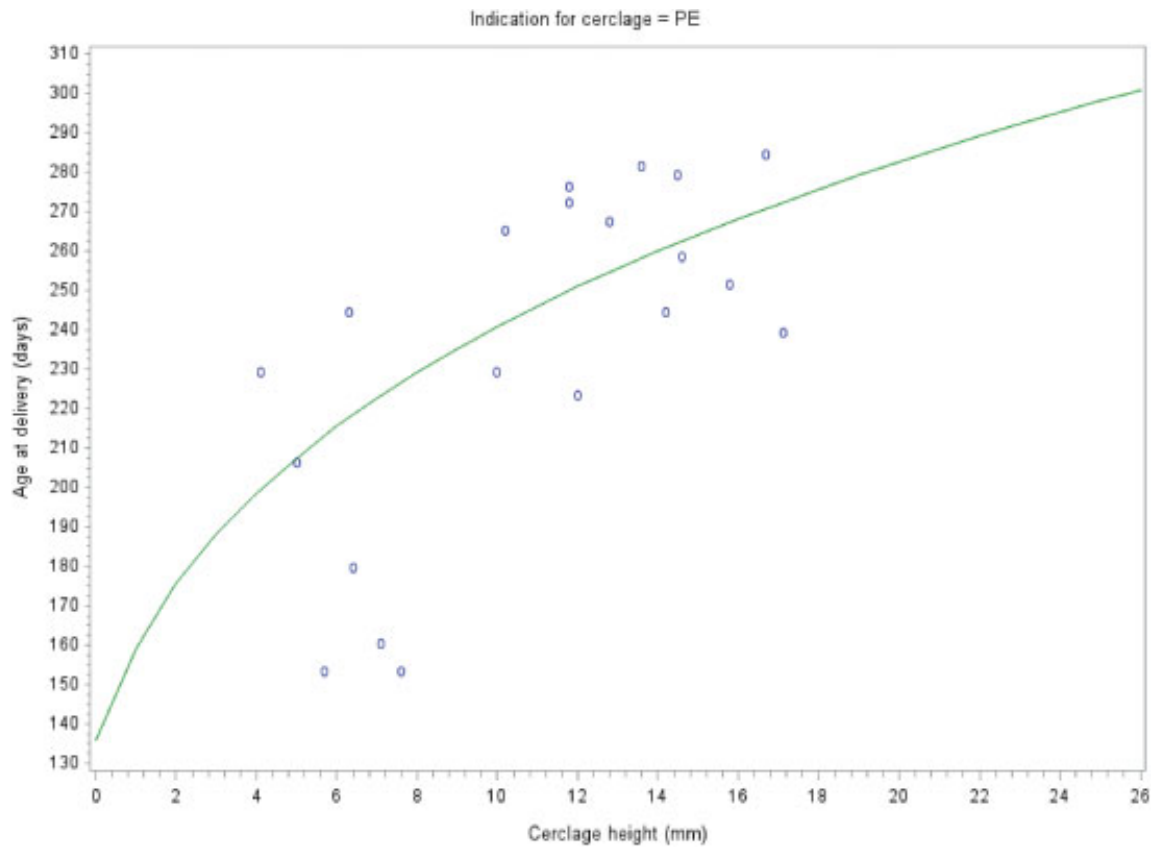

Fig. 5 The curvilinear regression function (adjusted for mean cervical length for cases with that indication), overlaid over raw data points for History indicated cerclages.

function (adjusted for mean CL for cases with that indication) for each indication, overlaid over raw data points.

Logistic regression predicting delivery $\leq 34$ weeks ( $n=29$ early deliveries) produced similar results; the CerH effect was significant for history indicated (pseudo$R^{2}=0.31, p=0.002$ ) and physical exam indicated (pseudo$\left.R^{2}=0.71, p=0.024\right)$, but not ultrasound indicated cerclages (pseudo- $R^{2}=0.34, p=0.090 ;$-Figs. 5 and 6).

There is modest evidence (based on a small subsample) that age at delivery is positively associated with cerH for cases where the indication for cerclage is physical exam. Though there is also statistical significance for the history indicated group, it is not clinically significant. For sonogram indicated the association is (if nonzero), is possibly much weaker.

\section{Discussion}

We found that when a cerclage is placed for a dilated cervix or a poor obstetrical history, the location of the stitch may influence the age at which the fetus is delivered. Specifically,

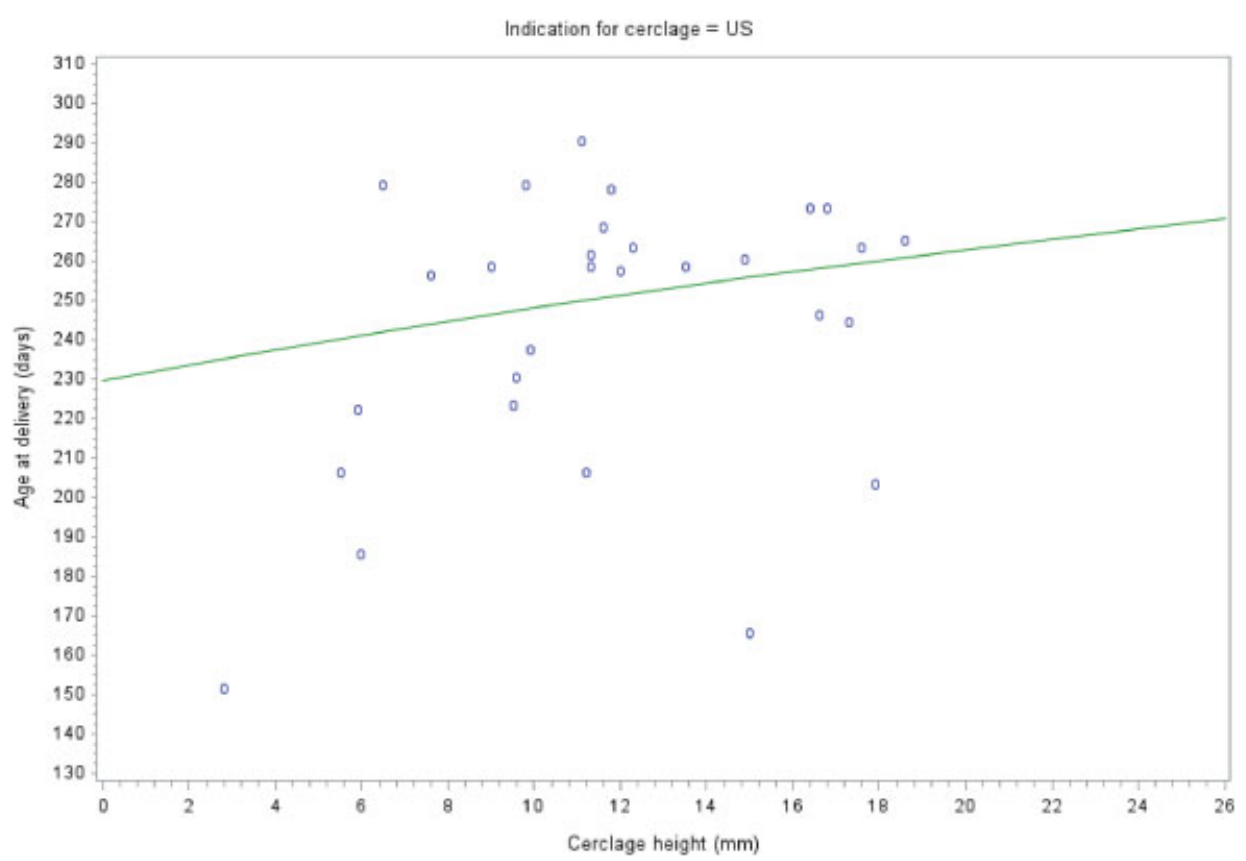

Fig. 6 The curvilinear regression function (adjusted for mean cervical length for cases with that indication), overlaid over raw data points for Ultrasoud indicated cerclages. 
the higher the cerclage stitch is placed, the more advanced the gestational age at delivery. This is statistically significant for both groups but likely only clinically significant in the group with the dilated cervix. We did not see similar associations for cerclages placed for ultrasound indications.

Cervical cerclage is a technique used to prolong pregnancy in patients with a clinical diagnosis of cervical insufficiency. Prior studies have compared the different techniques used for cerclage placement and found an increase in cerH of approximately $2.7 \mathrm{~mm}$ with Shirodkar versus McDonald. 5,6 However, it was felt at the time that this slight gain did not justify exposing the patient to increased risks given no difference in prognosis or delivery time between them. ${ }^{9}$ Other studies have assessed the relationship between cerclage position as seen on transvaginal sonogram and preterm delivery and have found no relationship ${ }^{10}$; and some more recent investigations have studied the relationship between cerH and preterm delivery in ultrasound-indicated cerclages only, and found no relationship. ${ }^{11}$

Thus, there is still uncertainty about whether height influences outcomes. To explore this issue further, we performed a retrospective cohort study of cerclages placed at one institution (MMC). To our knowledge, (using search Google Scholar and PubMed as search engines, with search terms, "age at delivery" and "cerclage height") this is the first study analyzing cerclages placed for all the ACOG recognized indications, and addressing the relationship of cerclage location with gestational age at delivery.

Prior research has questioned the utility of history indicated cerclage ${ }^{12}$ by suggesting that up to $50 \%$ of patients that receive a history indicated cerclage will deliver after 37 weeks without any intervention. Thus, these patients may represent a mix of women with and without a true indication for cerclage. Our results show that for some women with history indicated cerclage, position may be directly and positively related to gestational age at delivery, suggesting that at least for some, the procedure, if done properly can have a salutary effect.

Our data also suggest that in patients with physical exam indicated cerclages, trying to achieve a greater cerH (by, for example, retrograde filling the bladder, preoperative amnioreduction, placement of an intrauterine Foley's catheter) might be associated with a prolongation of pregnancy. This could be clinically significant, and may indicate the need for more aggressive techniques for higher stitch placement.

We, in agreement with previous reports, ${ }^{11}$ did not find an association in patients with ultrasound indicated cerclage. One possible explanation for this finding is that the small sample size resulted in a bias toward the null. It is possible that a sufficiently large subset of these patients is not helped by cerclage, masking our ability to show a benefit among the others.

\section{Limitation}

We do need to acknowledge some limitations in our study. There was a variation in time between stitch placement and subsequent ultrasound (1-4 weeks). Though this variation may have made the PRL measurement unreliable as a predictor since that measurement can change over time as a cervix shortens, it is unlikely that CerH and CL were influenced, since these measurements are distal to a static stitch. Interestingly, our data shows $\mathrm{CL}$ is not a predictor of age at delivery. This may support the practice of not checking routine $\mathrm{CL}$ measurements after cerclage placement.

\section{Conclusion}

In conclusion, in women with a cerclage performed for a dilated cervix, our results suggest that obstetricians should try to place the stitch as high as is safely possible. That may also be the case in women with a history indicated cerclage. The advantage of a higher stich is less clear in other settings.

Conflict of Interest

None.

\section{References}

1 American College of Obstetricians and Gynecologists' Committee on Practice Bulletins-Obstetrics. Practice Bulletin No. 171: Management of Preterm Labor. Obstet Gynecol 2016;128(04):e155-e164

2 March of dimes prematurity campaign: activities and milestones. Available at: https://www.marchofdimes.org/mission/march-ofdimes-prematurity-campaign.aspx. Accessed May 30, 2017

3 American College of Obstetricians and Gynecologists'. Practice bulletin no 142: cerclage for the management of cervical insufficiency. Obstet Gynecol 2014;123(2, Pt. 1):372-379

4 Robert R, Creasy RK, Iams JD, Lockwood CJ, Moore T, Greene MF. Creasy and Resnik's Maternal-Fetal Medicine: Principles and Practice, 7th edition. Philadelphia, PA: Elsevier Inc.; 2014:658660

5 Odibo A, To M, Berghella V, Rust O, Althuisius S. Shirodkar versus Mcdonald cerclage for the prevention of preterm birth (PTB) in women with short cervical length. AJOG 2005;193(06):S155

6 McDonald IA. Suture of the cervix for inevitable miscarriage. J Obstet Gynaecol Br Emp 1957;64(03):346-350

7 Guzman ER, Houlihan C, Vintzileos A, Ivan J, Benito C, Kappy K. The significance of transvaginal ultrasonographic evaluation of the cervix in women treated with emergency cerclage. Am J Obstet Gynecol 1996;175(02):471-476

8 Scheib S, Visintine JF, Miroshnichenko G, Harvey C, Rychlak K, Berghella V. Is cerclage height associated with the incidence of preterm birth in women with an ultrasound-indicated cerclage? Am J Obstet Gynecol 2009;200(05):e12-e15

9 Mella MT, Berghella V. Prediction of preterm birth: cervical sonography. Semin Perinatol 2009;33(05):317-324

10 Rozenberg P, Sénat MV, Gillet A, Ville Y. Comparison of two methods of cervical cerclage by ultrasound cervical measurement. J Matern Fetal Neonatal Med 2003;13(05):314-317

11 Melle L, Le Ray C, Delorme P, Anselem O, Goffinet F, Marcellin L. [Does post operative sonographic position of preventive cervical cerclage affect gestational age at birth?] Gynécol Obstét Fertil 2016;44(12):679-684

12 Scheib S, Visintine JF, Miroshnichenko G, Harvey C, Rychlak K, Berghella V. Is cerclage height associated with the incidence of preterm birth in women with an ultrasound-indicated cerclage? Am J Obstet Gynecol 2009;200(05):e12-e15

13 Suhag A, Reina J, Sanapo L, et al. Prior ultrasound-indicated Cerclage: Comparison of cervical length screening or History indicated cerclage in the next pregnancy. Obstet Gynecol 2015; 126(05):962-968 